\section{Você Pensa em Infarto Muscular no Paciente Diabético?}

\section{RESUMO}

O infarto muscular diabético (IMD) é uma complicação incomum do diabetes (DM) de longa duração. Esta condição pode ocorrer em pacientes com DM tipo 1 ou 2 mal controlados, com presença de microangiopatia. O quadro clínico é de dor aguda e intensa com edema do músculo afetado, que persiste por muitas semanas e tem melhora espontânea. Apesar de incerta, a etiologia é atribuída à microangiopatia com oclusão das pequenas artérias. É diagnosticado por biópsia, embora os achados em T2 na ressonância magnética sejam típicos. O tratamento de escolha é analgesia apropriada, repouso no leito e cuidadoso controle metabólico. Relatamos 3 casos de IMD admitidos em um hospital geral que não foram de imediato diagnosticados, levando a conduta e tratamentos inadequados. Enfatizamos os aspectos clínico, de imagem e histológico do IMD, permitindo o diagnóstico precoce desta condição incomum, evitando tratamento inapropriado. (Arq Bras Endocrinol Metab 2004;48/4:559-563)

Descritores: Infarto muscular; Diabetes mellitus; Microangiopatia; Edema muscular; Dor

\begin{abstract}
Muscle Infarction: Do You Think About It in A Diabetic Patient?

Diabetic muscle infarction (DMI) is an uncommon complication of long standing diabetes (DM). This abnormal condition may occur in poorly controlled patients with type 1 and 2 DM with established microangiopathy. Clinical presentation is usually acute with severely painful swelling of the affected muscle, which persists for many weeks and has spontaneous recovery. Albeit uncertain, its etiology is associated with microangiopathy with occlusion of small arteries. This condition is diagnosed by biopsy although results from T2 - weight magnetic resonance are typical. Pain management, bed rest and careful metabolic control are the treatment of choice. We report 3 cases of DMI admitted to a general hospital who were initially misdiagnosed and led to inadequate treatment and management at onset of the condition. We emphasize the clinical, image and histological aspects of DMI in order to allow early awareness of this uncommon condition, avoiding unnecessary delay as well as hastening appropriate treatment. (Arq Bras Endocrinol Metab 2004;48/4:559-563)
\end{abstract}

Keywords: Muscle infarction; Diabetes mellitus; Complication; Microangiopathy; Swelling muscle; Pain

$\mathbf{O}$ INFARTO MUSCULAR DiabÉTICO (IMD) é uma complicação incomum do diabetes mellitus (DM). Apesar de descrito pela primeira vez em 1965 por Angervall e Stener (1), extenso artigo de revisão (2) relaciona apenas 115 casos publicados na literatura mundial, não tendo sido referi- apresentação de

caso

Nenza B. C. de Araújo

Nilton C. de Araujo Filho

Aida Begami Leal

Nair F. Rey Cardoso

Nilson Duarte Correa
Setor de Endocrinologia da Clínica

Médica (NBCA, NFRC, NDC) e Serviço de Reumatologia

(NCAF, ABL) do Hospital

Geral de Bonsucesso, Ministério da

Saúde, Rio de Janeiro, RJ. 
do até o momento nenhum caso em nosso meio. A apresentação clínica típica é de dor muscular aguda em membros inferiores (geralmente coxa), com aumento de volume do músculo afetado, em diabéticos tipo 1 ou 2, mal controlados e com complicações crônicas (3). A dor é intensa, piorando com os movimentos. Tem evolução auto limitada, mas recorrências no mesmo membro ou no contra lateral ocorrem na metade dos casos. O diagnóstico de certeza é histológico, ainda que a maioria dos autores considere suficiente o quadro clínico associado aos achados da ressonância magnética $(\mathrm{RM})(2,4,5)$.

Descrevemos 3 casos de IMD admitidos em um hospital geral entre os anos de 2000 e 2001 , que, apesar de típicos, não foram inicialmente diagnosticados e, por isso, tratados inadequadamente. Enfatizamos os aspectos clínico, laboratorial e de imagem da patologia, importantes para o diagnóstico diferencial.

\section{APRESENTAÇĀO DOS CASOS}

\section{Caso 1}

Sexo feminino, 48 anos, com DM tipo 2 e hipertensa há 15 anos, foi admitida na Emergência com dor intensa de início súbito e espontâneo, na face anteromedial da coxa direita e joelho homolateral. Referia quadro semelhante há 3 meses de menor intensidade com resolução espontânea. Controle irregular do DM com história de descompensação aguda há 4 meses.

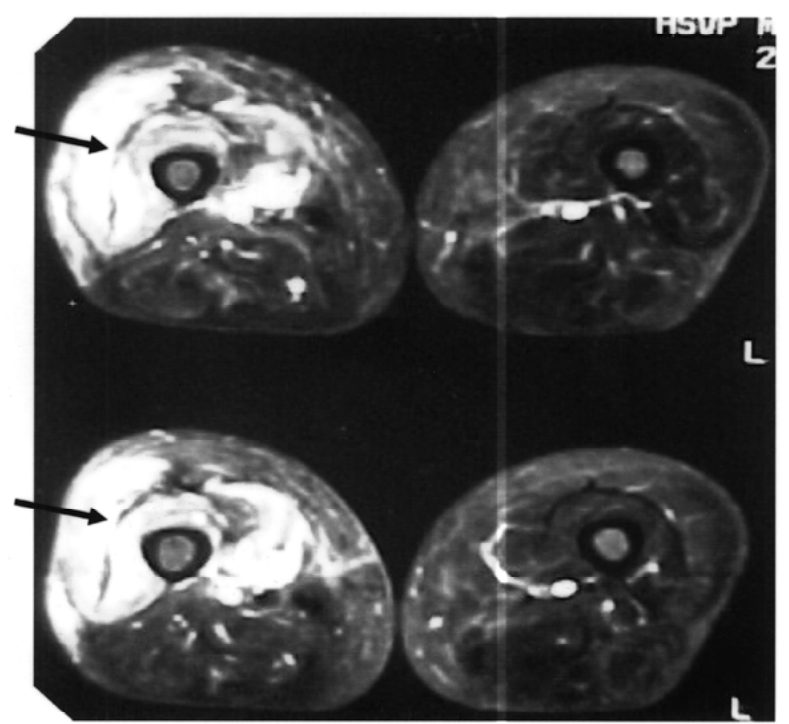

Figura 1. Caso 1. RM - corte transversal das coxas mostrando aumento de volume da direita com área focal de sinal mais intenso em T2 e hiposinal em T1 de permeio ao músculo vasto medial.

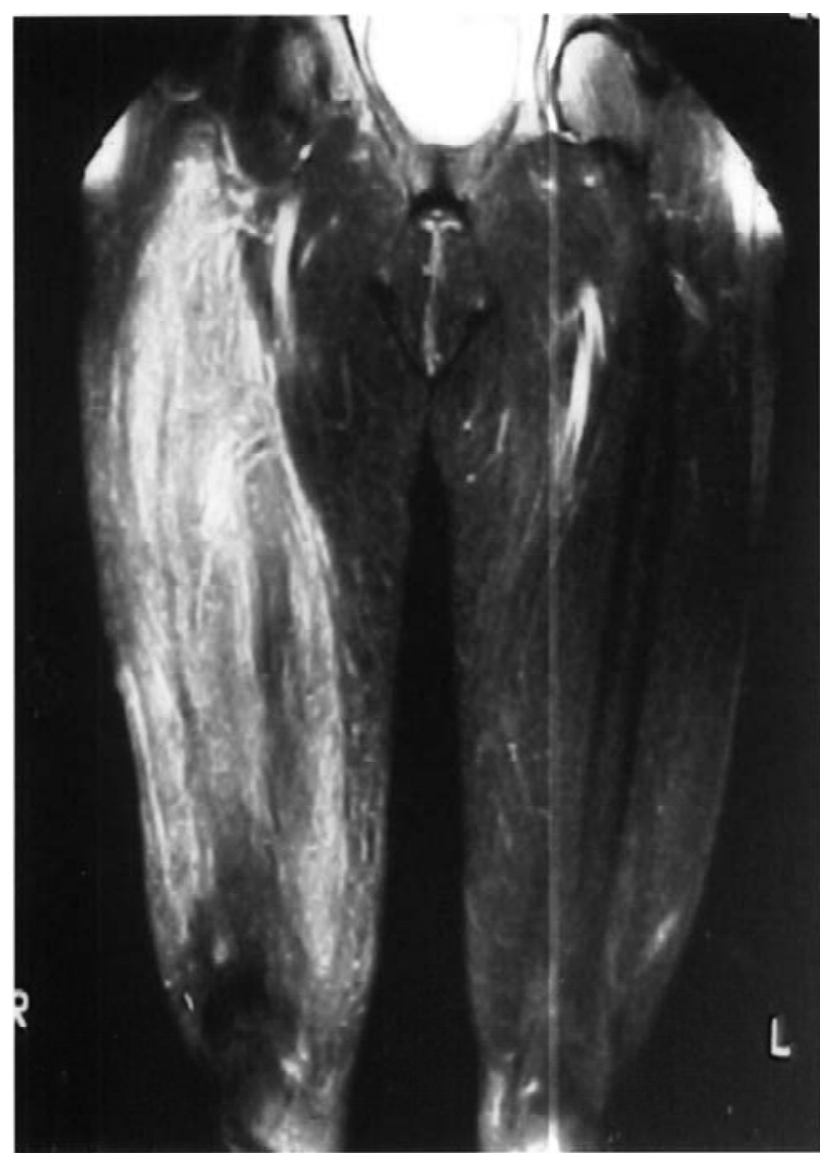

Figura 2. Caso 1. RM - corte longitudinal alteração do sinal dos músculos vastos direitos, estendendo-se da raiz da coxa ao joelho.

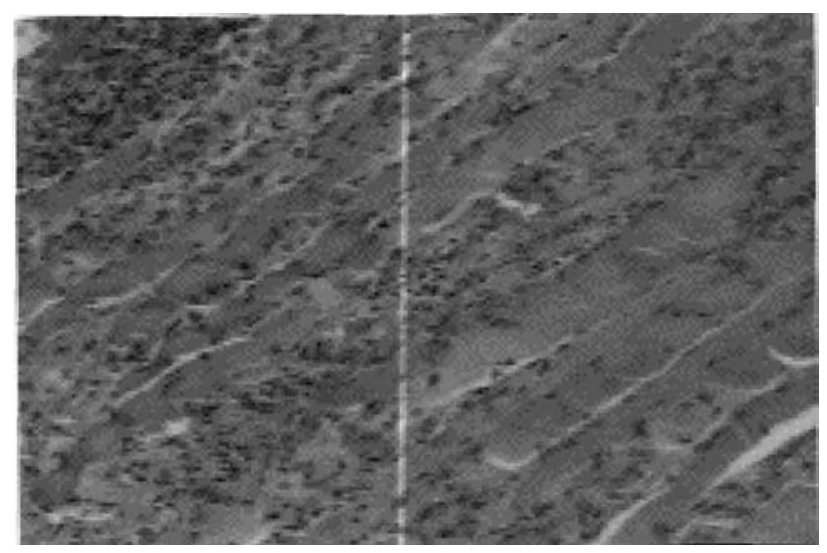

Figura 3. Caso 1. Fragmento de músculo esquelético com necrose hialina de algumas fibras, denso infiltrado inflamatório predominantemente mononuclear perivascular e focos hemorragia recente. 
Vinha em uso de metformina, glibenclamida e nifedipina, além de medicação para glaucoma. Transferida para a Clínica Reumatológica para investigação, apresentava exame físico normal exceto por mucosas hipocoradas $+/ 4+$, PA $170 \times 110 \mathrm{mmHg}$, taquicardia (110 batimentos/min) e aumento do volume da região interna da coxa e do joelho direitos, sem outros sinais inflamatórios.

\section{DIAGNÓSTICO INICIAL: Infecção de partes} moles e artrite a esclarecer. Iniciado antibioticoterapia e intensificado controle metabólico com insulina.

Os exames complementares mostraram leucometria de $10.200 \mathrm{~mm}^{3}$ sem desvio para a esquerda, hemoglobina: $10,3 \mathrm{mg} / \mathrm{dl}$, hematócrito: $32 \%$, glicemia: $260 \mathrm{mg} / \mathrm{dl}$, creatinina: $3,3 \mathrm{mg} / \mathrm{dl}$, urocultura e hemoculturas negativas, CPK: $144 \mathrm{U} / 1$ (VR: 26-174U/1), ultra-sonografia da coxa direita com aumento de partes moles e ausência de coleções ou alterações musculares. Aventada a hipótese de IMD. A RM da coxa revelou extensa alteração do sinal dos músculos vastos, estendendo-se da raiz da coxa ao joelho, área focal de sinal mais intenso em T2 de permeio ao músculo vasto medial direito com infiltração da gordura adjacente.

\section{BIÓPSIA MUSCULAR:}

Necrose hialina, focos de hemorragia recente, denso infiltrado perivascular e permeando a parede de vasos de pequeno calibre. Tratada, então, com analgesia vigorosa (opióides), repouso no leito e controle glicêmico. Evoluiu com recuperação lenta tendo alta 11 dias

\section{depois.}

\section{Caso 2}

Sexo masculino, 51 anos, com diagnóstico de DM tipo 2 há 10 anos, foi internado na Clínica de Nefrologia, onde fazia tratamento conservador da insuficiência renal, para investigação de dor de forte intensidade, contínua no $1 / 3$ inferior da coxa direita de aparecimento agudo alguns dias antes. Hipertenso de longa data, vinha em uso de hipoglicemiantes orais e hipotensores. Ao exame físico, apresentava mucosas hipocoradas $++/ 4+$, aumento do volume da coxa direita com região endurada, bem delimitada e dolorosa no terço inferior, derrame articular no joelho homolateral e edema perimaleolar discreto, bilateral, frio e indolor, sem outras alterações significativas. A PA era $180 \times 90 \mathrm{mmHg}$.

\section{DIAGNÓSTICO INICIAL: Processo infeccioso.}

\section{Prescrito antibiótico.}

Leucometria $23.400 \mathrm{~mm}^{3} \mathrm{sem}$ desvio para a esquerda, glicemia: $132 \mathrm{mg} / \mathrm{dl}, \mathrm{CPK}: 93 \mathrm{U} / \mathrm{l}$ (VR: 26-174U/l), uréia: $131 \mathrm{mg} / \mathrm{dl}$, creatinina: $2,3 \mathrm{mg} / \mathrm{dl}$, proteinúria: $7,82 \mathrm{~g} / 24 \mathrm{~h}$. Avaliado pela Reumatologia, sendo feita a suspeita de IMD. A cultura do líquido sinovial foi negativa. Ultra-sonografia da coxa mostrava hiperecogenicidade mal definida em planos musculares, sem coleções. Duplex scan arterial do membro inferior direito normal. RM com área focal de hipersinal em T2 de permeio ao músculo vasto medial direito no terço distal da coxa com infiltração da gordura subjacente.

\section{BIÓPSIA MUSCULAR:}

Necrose e degeneração muscular, fibras em regene-

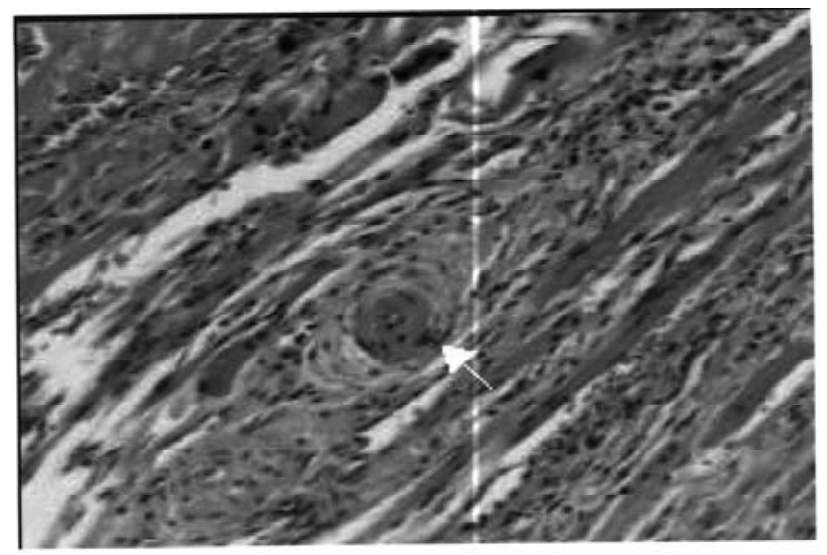

Figuras 4 e 5. Caso 2. Fragmento muscular exibindo necrose e degeneração de fibras, hemorragia focal. Algumas fibras em regeneração, espessamento arteriolar com edema do endotélio. Compatível com miopatia isquêmica. 
ração, espessamento arteriolar.

Tratado com analgesia vigorosa, repouso no leito e controle glicêmico, manteve dor importante com melhora após alguns dias e alta em 28 dias.

\section{Caso 3}

Sexo feminino, 68 anos, DM tipo 2 há 19 anos, com tratamento irregular. Internada na Emergência com massa dolorosa no terço inferior da coxa esquerda há 15 dias com dificuldade de deambular. Hipocorada, afebril, hiperglicêmica $(517 \mathrm{mg} / \mathrm{dl})$. Amaurótica à direita e hipertensa. Leucócitos: $11.400 \mathrm{~mm}^{3}$, HCT: 29,3\%, creatinina: $2,9 \mathrm{mg} / \mathrm{dl}$, uréia: $124 \mathrm{mg} / \mathrm{dl}$.

DIAGNÓSTICO INICIAL: Trombose Venosa Profunda. Iniciado heparinização e antibióticos. Ultra-sonografia da coxa esquerda com aumento de partes moles, sem evidências de trombose ou coleções. Feita, então, a suposição diagnóstica de IMD. Tomografia computadorizada da coxa esquerda: infiltração de celular subcutâneo e derrame articular do joelho do mesmo lado.

BIÓPSIA MUSCULAR: Miopatia com focos de regeneração. Vasculite de pequenos vasos. Retirada a heparina e os antibióticos. Feito repouso, analgesia vigorosa (tramadol e cetoprofeno) e controle glicêmico rigoroso. Evoluiu com dor intensa vários dias e teve alta com redução do volume da coxa e assintomática no $17^{\circ}$ dia de internação.

\section{DISCUSSĀO}

O infarto muscular é reconhecido como complicação rara, devendo ser considerado no diagnóstico diferencial

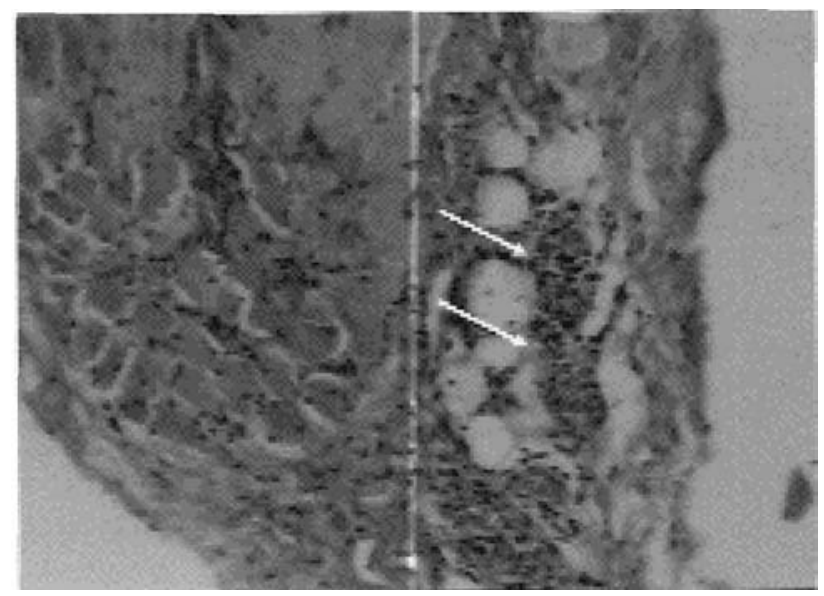

Figura 6. Caso 3. Fragmento de músculo com miopatia crônica inflamatória, focos de regeneração. Vasculite em vasos de pequeno calibre. das massas dolorosas em membros inferiores de pacientes com DM que mais freqüentemente são resultado de abcesso de tecidos moles, piomiosite, trombose venosa profunda e neoplasias musculares (6).

Com freqüência, pacientes com DM com esta condição recorrem inicialmente aos ortopedistas e reumatologistas por tratar-se de queixa músculoesquelética.

Os 3 casos descritos apresentavam as principais características clínicas do infarto muscular, ou seja, DM de longa duração (média de 14,6 anos), sem acompanhamento adequado (identificado na história e na terapêutica em uso), com sinais de complicação crônica, principalmente insuficiência renal (segundo a literatura, presente em $90 \%$ dos casos), e que desenvolveram dor intensa de início agudo com aumento de volume do músculo afetado (achados clínicos encontrados em $80 \%$ e $75,9 \%$, respectivamente). Na literatura revisada, os músculos da coxa são, na maioria, os atingidos ( $83,7 \%$ dos vastos medial e lateral) (2). O comprometimento articular, como nos casos 1 e 2, é incomum e, quando presente, é atribuído à dificuldade de drenagem venosa pelo aumento do músculo afetado, elevando a pressão hidrostática intra-articular, favorecendo o acúmulo de líquido sinovial (7).

Os achados laboratoriais não são específicos. As enzimas musculares podem estar elevadas ou normais em $52,7 \%$ dos casos, a leucocitose não é freqüente e a velocidade de hemossedimentação pode ser maior que $100 \mathrm{~mm} / \mathrm{h}$. A patogênese ainda não é clara e a hipótese mais aceita é que o infarto seja decorrente da microangipatia e edema tecidual secundário, levando ao aumento da pressão local com comprometimento do fluxo sangüíneo e conseqüente isquemia (8-10). Outro fator implicado é a alteração do sistema de coagulação-fibrinolise com hipercoagulabilidade e formação de microtrombos $(8,9)$.

O IMD pode ser diagnosticado pela apresentação clínica e os achados de imagem. Na experiência dos autores $(2,4,5)$, o que também verificamos, a RM é o exame de escolha, com os dados característicos de aumento difuso do grupo muscular afetado e perda parcial da gordura intermuscular e edema subcutâneo. $\mathrm{O}$ músculo infartado mostra hipersinal em T2-weighted comparado com os músculos adjacentes, conseqüente ao edema e alterações inflamatórias que acompanham o infarto $(2,12)$. A ultrassonografia é útil na diferenciação de abcessos, TVP e hematomas, embora alguns autores descrevam imagem intramuscular bem delimitada hipoecóica com características especiais, não encontrada em nossos casos (11). A TC pode apresentar, como no caso 3 , aumento difuso do músculo e da atenuação 
da gordura subcutânea. O diagnóstico de certeza, entretanto, é através da biópsia muscular por agulha, como proposto por alguns, ou aberta, como em nossos casos, que mostra grandes áreas de necrose de todos os elementos do músculo com infiltração de mononucleares ou polimorfonucleares, edema e variáveis graus de regeneração das miofibras com fibrose dependendo da idade da lesão $(5,12)$.

$\mathrm{O}$ caráter benigno e a resolução espontânea e lenta (6 semanas a 6 meses) dispensariam internação para alguns autores, entretanto, nos nossos casos, a intensidade da dor e a necessidade de afastar outras patologias obrigaram-nos a interná-los.

O tratamento desta condição clínica é com repouso no leito (o exercício agrava e prolonga o tempo de evolução), analgesia e cuidadoso controle metabólico $(4,14)$. O prognóstico é bom, mas as recidivas acontecem em cerca de $50 \%$ dos pacientes, como no caso 2 .

\section{CONCLUSÃO}

O retardo no diagnóstico de IMD em nossos casos levou ao uso desnecessário de antibióticos e anticoagulante (no caso 3), bem como a um prolongado tempo de internação. Apesar do quadro álgico importante e incapacitante, o infarto muscular é uma condição benigna e auto limitada. Embora raro, deve ser descartado em toda massa dolorosa muscular com CK normal ou elevada e com aumento de sinal em T2 na RNM, sendo a biópsia muscular necessária ao diagnóstico na impossibilidade de realização daquele método de imagem ou nos casos de apresentação atípica ou progressão da condição.

\section{AGRADECIMENTOS}

À professora Dra. Marília de Brito Gomes, chefe da disciplina de Diabetes e Metabologia da UERJ, e ao professor Dr. Maurício de Andrade Pérez, professor assistente de Epidemiologia da UFRJ, pela colaboração e incentivo.

\section{REFERÊNCIAS}

1. Angervall L, Sterner B. Tumoriform focal muscular degeneration in two diabetic patients. Diabetologia 1965; 1:39-42.
2. Trujillo-Santos AJ. Diabetic muscle infarction: An underdiagnosed complication of long standing diabetes. Diabetes Care 2003;26:211-5.

3. Melikian N, Bingham J, Goldsmith DJ. Diabetic muscle infarction: An unusual cause of acute limb swelling in patients on hemodialysis. Am J Kidney Dis 2003;41:13226.

4. Chason DP. Diabetic muscle infarction. Skeletal Radiol 1996;25:127-32.

5. Grigoriadis E, Fam AG, Starok M, Ang LC. J Rheumatol 2000;27:1063-8.

6. Palmer GW, Greco TP. Diabetic thigh muscle infarction in association with antiphospholipid antibodies. Semin Arthritis Rheum 2001;30:272-80.

7. Yoo WH, Kun CH, Park JH, Kim JR, Park TS, Balk HS. Case report: Diabetic muscle infarction presenting as knee arthralgia. Rheumatol Int 2001;21:36-9.

8. Dellis S, Cianco G, Casillas J, Figueiro J, Garcia A, Miller $\mathrm{S}$, et al. Diabetic muscle infarction after simultaneous pancreas-kidney transplant. Clin Transplant 2002; 16:295-300.

9. Scully RE, Mark EJ, McNelly WF, Ebeling SH, Phillips LD. Case 29-1997. Case records of Massachusetts General Hospital. N Engl J Med 1997;337:839-45.

10. Silberstein L, Button KE, Marsh FP, Raftery MJ, D'Cruz D. An unexpected case of muscle pain in diabetes. Ann Rheum Dis 2001;60:310-2.

11. Delaney-Sathy LO, Fessell DP, Jacobson JA, Hayes CW. Sonography of diabetic muscle infarction with MR imaging, CT, and pathologic correlation. Am J Roentgenol 2000;174:165-9.

12. Jelinek JS, Murphey MD, Aboulafia AJ, Dussault RG, Kaplan PA, Snearly WN. Muscle infarction in patients with diabetes mellitus. MR Imaging Findings Radiology 1999;211:241-7.

13. Chester CS, Banker $B Q$. Focal infarction of muscle in diabetics. Diabetes Care 1986;9:623-30.

14. Pamoukian VN, Rubino F, Iraci JC. Review and case report of idiopathic lower extremity compartment syndrome and its treatment in diabetic patients. Diabetes Metab 2000;26:489-92.

Endereço para correspondência:

Neuza Braga Campos de Araújo

Rua Mário Barreto 66, ap. 302

20510-390 Rio de Janeiro, RJ

Fax: (21) 2570-9658

e-mail: russarj@terra.com.br 